Estudo sobre as temperaturas médias em Campinas

\title{
HERNANI GODOY
}

Instituto Agronômico do Estado, em Campinas.

IZAIAS R. NOGUEIRA E FREDERICO PIMENTEL GOMES

E. S. A. "Luiz de Queiroz". 


\section{INTRODUÇÃO}

Neste trabalho são estudados registros de temperatura de 67 anos, correspondentes ao período de 1890 a 1956, referentes à cidade de Campinas (Estado de S. Paulo, Brasil). Além das médias anuais, investigaram-se detalhadamente as médias' dos meses de janeiro, abril, julho e outubro, como representativos das quatro estações do ano, tendo-se em vista especialmente a descoberta de tendências seculares, pelo método dos polinômios ortogonais.

As temperaturas foram determinadas por termômetros de mercúrio (R. Fuess), de fabricação alemã, cam graduação de dois em dois décimos de grau centígrado. As temperaturas médias diárias foram sempre determinadas a partir das leituras às 7 horas $\left(t_{7}\right)$, às 14 horas $\left(t_{14}\right)$ e às 21 horas $\left(t_{21}\right)$ pela fórmula:

$$
\text { Temperatura média }=\frac{t_{7}+t_{14}+2 t_{21}}{4} .
$$

De 1890 a 1931 as observações se fizeram sempre no mesmo local do Instituto Agronômico de Campinas; em 1931 o posto meteorológico foi transportado para sítio 100 metros adiante, onde permanece até hoje.

\section{TEMPERATURAS MEDIAS ANUAIS}

Pelo método dos polinômios ortogonais foram tentadas equações de regressão até o $4 .^{\circ}$ grau. A análise da variância, que consta do quadro n. ${ }^{\circ} 1$, demonstra a existência de compontes significantes de primeiro e segundo grau.

Note-se que indicamos com um asterisco a significação ao nível de $5 \%$ de probabilidade, com dois, ao nível de $1 \%$, e com três, ao de $0,1 \%$.

ANÁLISE DA VARIÂNCIA DAS TEMPERATURAS MEDIAS ANUAIS

\begin{tabular}{l||c|c|c}
\hline \multicolumn{1}{c|}{ Causa de Variação } & $\begin{array}{c}\text { Graus de } \\
\text { liberdade }\end{array}$ & $\begin{array}{c}\text { Soma de } \\
\text { Quadrados }\end{array}$ & $\begin{array}{c}\text { Quadrado } \\
\text { Médio }\end{array}$ \\
\hline Regressão linear $\ldots \ldots \ldots$ & 1 & 7,19 & $7,19 * * *$ \\
Regressão quadrática $\ldots \ldots$ & 1 & 2,55 & $2,55 * *$ \\
Regressão cúbica $\ldots \ldots \ldots$ & 1 & 0,87 & 0,87 \\
Regressão de $4 .^{\circ}$ grau $\ldots$. & 1 & 0,14 & 0,14 \\
Resíduo $\ldots \ldots \ldots \ldots \ldots \ldots$ & 62 & 15,52 & 0,25 \\
\hline
\end{tabular}


A equação de regressão obtida é:

$$
y=19,95-0,0219 x+0,00057 x^{2},
$$

onde $y$ é a temperatura èm graus centígrados e $x$ indica o número de anos decorridos a partir de 1889; assim para $1890, x=1$, para $1891, x=2$, etc.

Essa equação indica que houve uma tendência de queda de temperatura média anual de 1890 até $1908(x=19)$, daí por diante surgindo uma tendência para elevação dessa temperatura como mostram os dados calculados seguintes.

TEMPERATURAS MÉDIAS ANUAIS DE CAMPINAS (SP) CALCULADAS PELA EQUAÇÃO DE REGRESSÃO

$\begin{array}{cc}\text { Ano } & \begin{array}{c}\text { Temperatura Média Anual } \\ \text { (Graus centígrados) }\end{array} \\ 1890 & 19,93 \\ 1900 & 19,78 \\ 1908 & 19,74 \text { (mínima) } \\ 1910 & 19,75 \\ 1920 & 19,82 \\ 1930 & 20,01 \\ 1940 & 20,32 \\ 1950 & 20,74 \\ 1956 & 21,05\end{array}$

Deixando de lado a regressão, a média das temperaturas médias anuais em 67 anos foi, em Campinas; igual a $20,08^{\circ} \mathrm{C}$ com êrro padrão da média igual a $0,08^{\circ} \mathrm{C}$.

$\mathrm{O}$ intervalo de confiança, ao nível de $5 \%$ de probabilidade, vai de $19,92^{\circ} \mathrm{C}$ a $20,24^{\circ} \mathrm{C}$.

\section{TEMPERATURAS MÉDIAS DE JANEIRO}

A análise da variância consta do quadro n.o 2. 
ANÁLISE DA VARIÂNCIA DAS TEMPERATURAS MÉDIAS DE JANEIRO

\begin{tabular}{l||c|c|c}
\hline Causa de Variação & $\begin{array}{c}\text { Graus de } \\
\text { liberdade }\end{array}$ & $\begin{array}{c}\text { Soma de } \\
\text { Quadrados }\end{array}$ & $\begin{array}{c}\text { Quadrado } \\
\text { Médio }\end{array}$ \\
\hline Regressão linear $\ldots \ldots \ldots$ & 1 & 7,14 & $7,14 * *$ \\
Regressão quadrática $\ldots \ldots$ & 1 & 11,19 & $11,19 * *$ \\
Regressão cúbica $\ldots \ldots \ldots$ & 1 & 0,04 & 0,04 \\
Regressão $4 .^{*}$ grau $\ldots \ldots$. & 1 & 1,38 & 1,38 \\
Resíduo $\ldots \ldots \ldots \ldots \ldots \ldots$ & 62 & 43,28 & 0,69 \\
\hline
\end{tabular}

QUADRO N. 2

Também neste caso são significativos os componentes de $1 .^{\circ}$ e $2 .^{\circ}$ grau, com equação de regressão

$$
\mathrm{y}=23,08-0,0661 \mathrm{x}+0,00122 \mathrm{x}^{2},
$$

onde, como no caso anterior, $x$ é o número de anos decorridos a partir de 1889. Esta equação indica uma tendência de queda nas temperaturas médias de janeiro, de 1890 até 1916, seguida de tendência de elevação progressiva daí por diante, como se verifica, aliás, pelos dados seguintes.

TEMPERATURAS MEDIAS DE JANEIRO, EM CAMPINAS (SP), CALCULADAS PELA EQUAÇÃO DE REGRESSÃO

$\begin{array}{cc}\text { Ano } & \begin{array}{r}\text { Temperatura Média de Janeiro } \\ \text { (Graus centígrados) }\end{array} \\ 1890 & 23,02 \\ 1900 & 22,50 \\ 1910 & 22,23 \\ 1916 & 22,19 \text { (mínima) } \\ 1920 & 22,20 \\ 1930 & 22,42 \\ 1940 & 22,88 \\ 1950 & 23,59 \\ 1956 & 24,13\end{array}$

Deixada de lado a regressão, a média dos 67 anos foi $22,70^{\circ} \mathrm{C}$, com êrro padrão da média $0,12^{\circ} \mathrm{C}$. 
O intervalo de confiança da média ao nível de $5 \%$ de probabilidade vai de $22,46^{\circ} \mathrm{C}$ a $22,94^{\circ} \mathrm{C}$.

\section{TEMPERATURAS MEDIAS DE ABRIL}

A análise da variância consta do Quadro n.o 3.

ANÁLISE DA VARIÂNCIA DAS TEMPERATURAS MEDIAS DE ABRIL

\begin{tabular}{l||c|c|c}
\hline Causa de Variação & $\begin{array}{c}\text { Graus de } \\
\text { liberdade }\end{array}$ & $\begin{array}{c}\text { Soma de } \\
\text { Quadrados }\end{array}$ & $\begin{array}{c}\text { Quadrado } \\
\text { Médio }\end{array}$ \\
\hline \hline Regressão linear $\ldots \ldots \ldots$ & 1 & 2,81 & 2,81 \\
Regressão quadrática $\ldots \ldots$ & 1 & 0,72 & 0,72 \\
Regressão cúbica $\ldots \ldots \ldots$ & 1 & 2,69 & 2,69 \\
Regressão de $40^{\circ}$ grau $\ldots$. & 1 & 0,29 & 0,29 \\
Resíduo $\ldots \ldots \ldots \ldots \ldots \ldots$ & 62 & 66,38 & 1,07 \\
\hline
\end{tabular}

QUADRO N. 3

Neste caso não há significação para nenhum dos componentes de regressão isolados.

A média dos 67 anos foi $20,42^{\circ} \mathrm{C}$, com êrro padrão da média $0,13^{\circ} \mathrm{C}$.

$O$ intervalo de confiança a $5 \%$ de probabilidade vai de 20,16 a $20,68^{\circ} \mathrm{C}$.

\section{TEMPERATURAS MEDIAS DE JULHO}

A análise da variância consta do quadro n. ${ }^{\circ} 4$.

ANÁLISE DA VARIÂNCIA DAS TEMPERATURAS MEDIAS DE JULHO

\begin{tabular}{l||c|c|c}
\hline \multicolumn{1}{c||}{ Causa de Variação } & $\begin{array}{c}\text { Graus de } \\
\text { liberdade }\end{array}$ & $\begin{array}{c}\text { Soma de } \\
\text { Quadrados }\end{array}$ & $\begin{array}{c}\text { Quadrado } \\
\text { Médio }\end{array}$ \\
\hline \hline Regressão linear $\ldots \ldots \ldots$ & 1 & 4,93 & $4,93 *$ \\
Regressão quadrática $\ldots \ldots$ & 1 & 2,44 & 2,44 \\
Regressão cúbica $\ldots \ldots \ldots$ & 1 & 0,18 & 0,18 \\
Regressão de $4 .^{\circ}$ grau $\ldots .$. & 1 & 0,67 & 0,67 \\
Resíduo $\ldots \ldots \ldots \ldots \ldots$. & 62 & 72,84 & 1,17 \\
\hline
\end{tabular}

QUADRO N.0 4 
Há, pois, significação ao nível de $5 \%$ para a regressão linear. A equação de regressão obtida é:

$$
\mathrm{y}=16,01+0,0140 \mathrm{x} .
$$

Houve, pois, tendência secular de elevação constante das temperaturas médias de julho, à taxa anual de 0,0140 grau centígrado. As médias calculadas seguintes dão idéia melhor dessa elevação.

TEMPERATURAS MEDIAS DE JULHO, EM CAMPINAS (SP), CALCULADAS PELA EQUAÇÃO DE REGRESSÃO

$\begin{array}{rr}\text { Ano } & \begin{array}{r}\text { Temperaturas Méd } \\ \text { (graus centígra }\end{array} \\ 1890 & 16,02 \\ 1900 & 16,16 \\ 1910 & 16,30 \\ 1920 & 16,44 \\ 1930 & 16,59 \\ 1940 & 16,73 \\ 1950 & 16,86 \\ 1956 & 16,95\end{array}$

Deixada de lado a regressão, a média dos 67 anos foi $16,49^{\circ} \mathrm{C}$, com êrro padrão da média $0,14^{\circ} \mathrm{C}$. O intervalo de confiança ao nível de $5 \%$ de probabilidade vai de $16,21^{\circ} \mathrm{C}$ a $16,77^{\circ} \mathrm{C}$.

\section{TEMPERATURA MÉDIAS DE OUTUBRO}

Análise da variância consta do quadro n. $0^{0} 5$.

ANÁlISE DA VARIÂNCIA DAS TEMPERATURAS MÉdIAS DE OUTUBRO

\begin{tabular}{|c|c|c|c|}
\hline Causa de Variação & $\begin{array}{l}\text { Graus de } \\
\text { liberdade }\end{array}$ & $\begin{array}{l}\text { Soma de } \\
\text { Quadrados }\end{array}$ & $\begin{array}{c}\text { Quadrado } \\
\text { Médio }\end{array}$ \\
\hline $\begin{array}{l}\text { Regressão linear } \ldots \ldots \ldots \\
\text { Regressão quadrática } \ldots \ldots \\
\text { Regressão cúbica } \ldots \ldots \ldots \\
\text { Regressão de } 4 .^{\circ} \text { grau } \ldots \\
\text { Resíduo } \ldots \ldots \ldots \ldots \ldots\end{array}$ & $\begin{array}{r}1 \\
1 \\
1 \\
1 \\
62\end{array}$ & $\begin{array}{r}7,07 \\
4,53 \\
1,76 \\
1,05 \\
66,38\end{array}$ & $\begin{array}{l}7,07 * \\
4,53 * \\
1,76 \\
1,05 \\
1,07\end{array}$ \\
\hline
\end{tabular}

QUADRO N. ${ }^{\circ} 5$ 
Neste caso só são significativos os componentes linear e quadrático. A equação obtida é:

$$
\mathrm{y}=20,55-0,0362 \mathrm{x}+0,00078 \mathrm{x}^{2} \text {. }
$$

Esta equação indica tendência de decréscimo de temperatura média de outubro de 1890 até 1912, daí por diante havendo tendência contrária.

Os dados seguintes são bem elucidativos.

TEMPERATURAS MEDIAS DE OUTUBRO, EM CAMPINAS (SP), CALCULADAS PELA EQUAÇAOO DE REGRESSAO

$\begin{array}{cc}\text { Ano } & \begin{array}{c}\text { Temperaturas Médias de Outu } \\ \text { (Graus centígrados) }\end{array} \\ 1890 & 20,52 \\ 1900 & 20,25 \\ 1910 & 20,14 \\ 1912 & 20,13 \text { (mínima) } \\ 1920 & 20,18 \\ 1930 & 20,38 \\ 1940 & 20,74 \\ 1950 、 & 21,25 \\ 1956 & 21,63\end{array}$

Deixada de lado a regressão, a média dos 67 anos foi $20,52^{\circ} \mathrm{C}$, com êrro padrão da média $0,14^{\circ} \mathrm{C}$.

O intervalo de confiança para êste mês, ao nível de $5 \%$ de probabilidade vai de $20,24^{\circ} \mathrm{C}$ a $20,80^{\circ} \mathrm{C}$.

\section{CONCLUSO์ES}

Em quase todos os casos estudados houve regressão significativa, de primeiro ou segundo grau, demonstrando tendência secular de modificação das temperaturas médias. foi

No que se refere à média anual, a equação de regressão

$$
y=19,95-0,0219 x+0,000057 x^{2}
$$


com $x$ indicando o número de anos decorridos a partir de 1889, sendo, pois, 1890 correspondente a $x=1,1891$; a $x=2$, etc. $(19,74)$.

Essa função tem mínimo correspondente ao ano de 1908

Para o mês de janeiro também se apresentou equação de regressão de segundo grau:

$$
y=23,08-0,0661 x+0,00122 x^{2},
$$

esta com mínimo em $1916\left(22,19^{\circ} \mathrm{C}\right)$.

Para o mês de abril não foi significativo nenhum componente de regressão.

Quanto ao mês de julho, a regressão obtida foi linear:

$$
y=16,01+0,0140 x
$$

o que indica tendência secular de aumento das temperaturas médias dêsse mês.

Finalmente, no que se refere a outubro, novamente se obteve equação de regressão de segundo grau:

$$
\mathrm{y}=20,55-0,0362 \mathrm{x}+0,00078 \mathrm{x}^{2},
$$

com mínimo em $1912\left(20,13^{\circ} \mathrm{C}\right)$.

A média anual determinada foi $20,08^{\circ} \mathrm{C}$, com intervalo de confiança, ao nível de $5 \%$ de probabilidade, de $19,92^{\circ} \mathrm{C}$ a $20,24^{\circ} \mathrm{C}$.

Para janeiro a média foi a $22,70^{\circ} \mathrm{C}$, com intervalo de confiança, ainda ao nível de $5 \%$ de probabilidade, de 22,46 a $22,94^{\circ} \mathrm{C}$.

Para abril a média foi de $20,42^{\circ} \mathrm{C}$, com intervalo de confiança de 20,16 a $20,68^{\circ} \mathrm{C}$.

Para julho a média foi de $16,49^{\circ} \mathrm{C}$, com intervalo de confiança de 16,21 a $16,7^{\circ} \mathrm{C}$.

Para outubro a média foi de $20,52^{\circ} \mathrm{C}$ e intervalo de confiança de 20,24 a $20,80^{\circ} \mathrm{C}$. 
Estudo sôbre as temperaturas...

DADOS DE TEMPERATURAS DE CAMPINAS (SP) TEMPERATURAS MEDIAS MENSAIS - 1890-1956

\begin{tabular}{|c|c|c|c|c|c|c|c|c|c|c|c|c|c|}
\hline ANOS & JAN. & PEV. & MAR. & ABR. & MAIO & JUкНО & JULHO & ACO. & 8BT. & ODT: & MOY. & DEZ. & $\begin{array}{l}\text { Médlas } \\
\text { Anuals }\end{array}$ \\
\hline $\begin{array}{l}1890 \\
1891 \\
1892 \\
1893 \\
1894 \\
1895 \\
1896 \\
1897 \\
1898 \\
1899 \\
1900 \\
1901 \\
1902 \\
1903 \\
1904 \\
1905 \\
1906 \\
1907 \\
1908 \\
1909 \\
1910 \\
1911 \\
1912 \\
1913 \\
1914 \\
1915 \\
1916 \\
1917 \\
1918 \\
1919 \\
1920 \\
1921 \\
1922 \\
1923 \\
1924 \\
1925 \\
1926 \\
1927 \\
1928 \\
1929 \\
1930 \\
1931 \\
1932 \\
1933 \\
1934 \\
1935 \\
1936 \\
1937 \\
1938 \\
1939 \\
1940 \\
1941 \\
1942 \\
1943 \\
1944 \\
1945 \\
1946 \\
1947 \\
1948 \\
1949 \\
1950 \\
1951 \\
1952 \\
1953 \\
1954 \\
1955 \\
1956\end{array}$ & 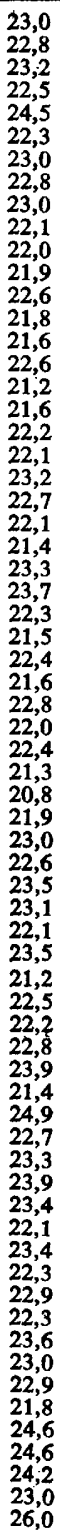 & 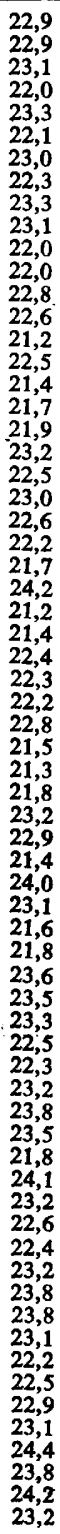 & 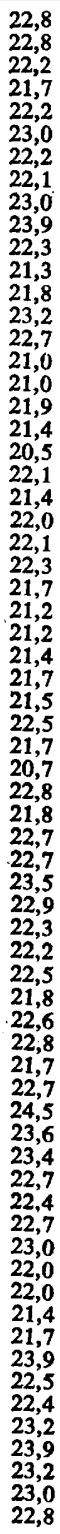 & 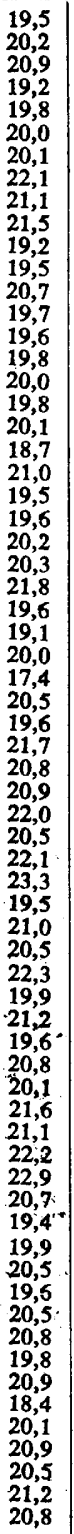 & 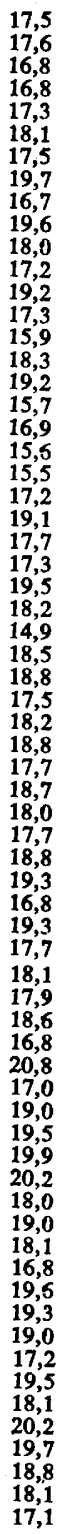 & 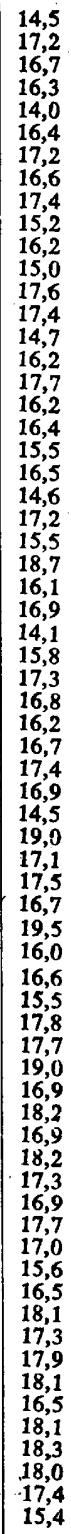 & 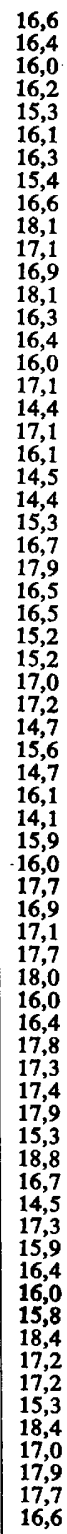 & 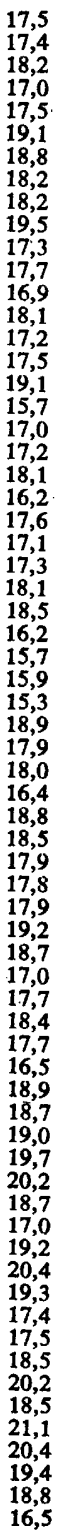 & 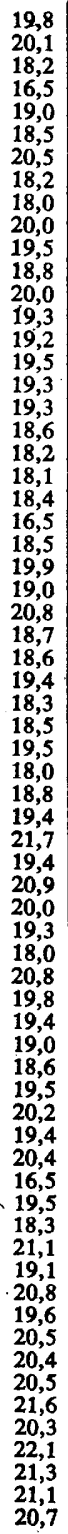 & 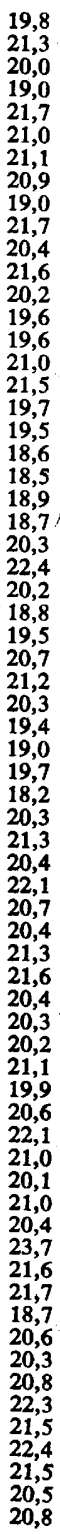 & 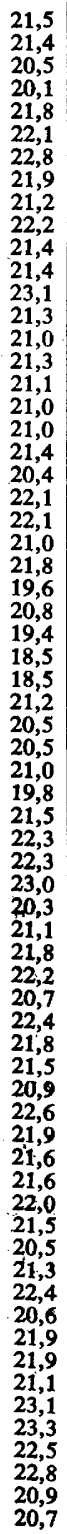 & 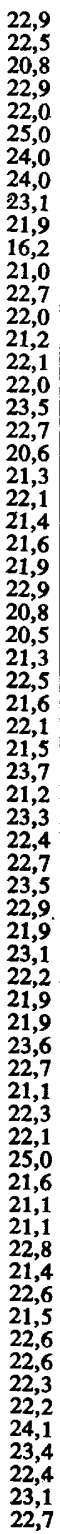 & 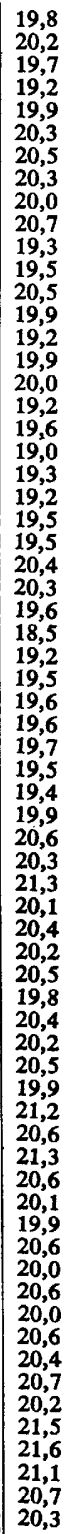 \\
\hline
\end{tabular}




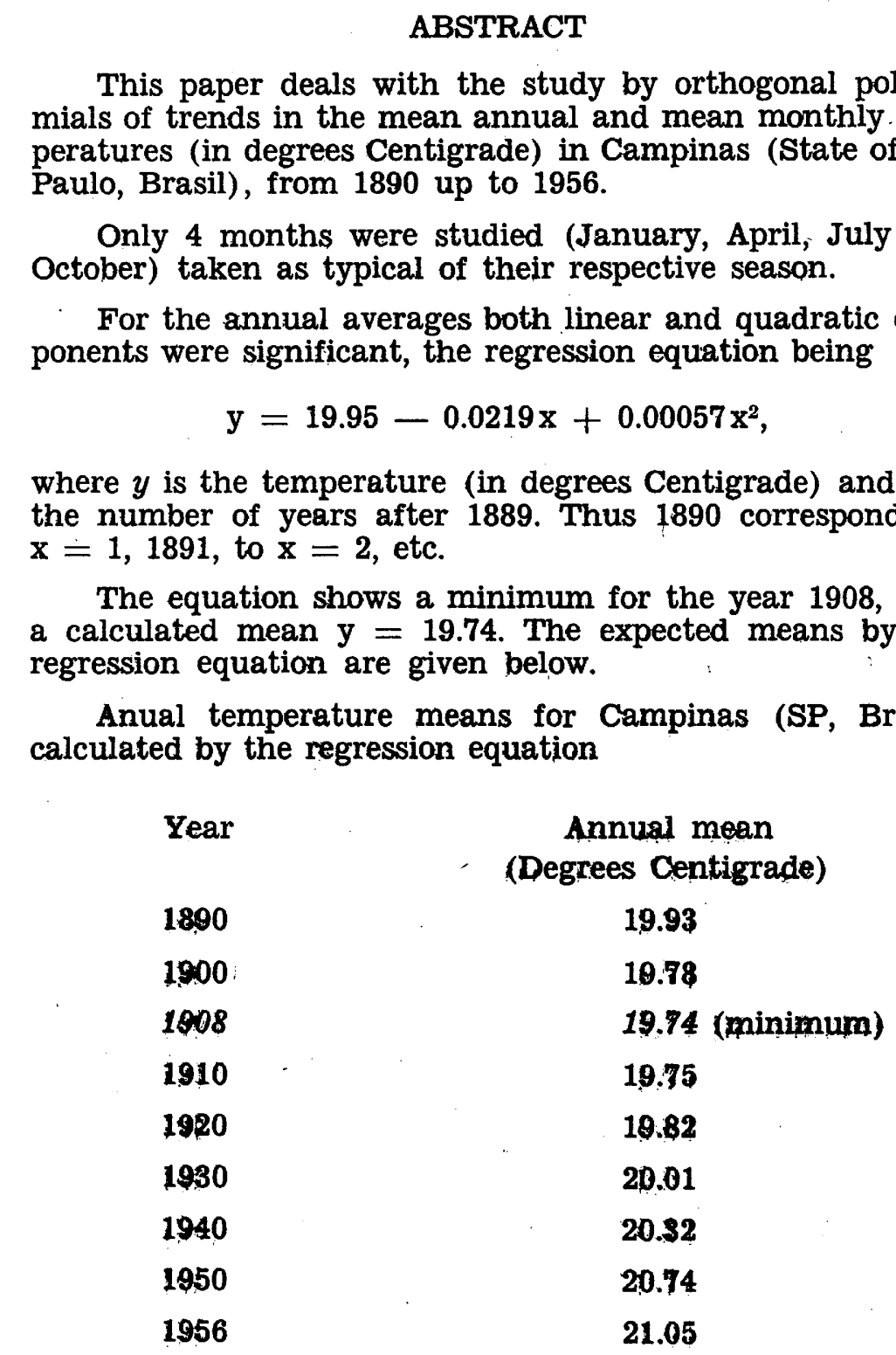

The mean for 67 years was $20.08^{\circ} \mathrm{C}$ with standard error of the mean $0.08^{\circ} \mathrm{C}$. 
For January the regression equation was

$$
\mathrm{y}=23.08-0.0661 \mathrm{x}+0.00122 \mathrm{x}^{2},
$$

with a minimum of $22.19^{\circ} \mathrm{C}$ for 1916. The average for 67 years was $22.70^{\circ} \mathrm{C}$, with standard error $0.12^{\circ} \mathrm{C}$.

For April no component of regression was significant. The average was $20.42^{\circ} \mathrm{C}$, with standard error $0.13^{\circ} \mathrm{C}$.

For July the regression equation was of first degree,

$$
\mathrm{y}=16.01+0.0140 \mathrm{x} \text {. }
$$

The average for 67 years was $16.49^{\circ} \mathrm{C}$, with standard error of the mean $0.14^{\circ} \mathrm{C}$.

Finally, for October the regression equation was

$$
\mathrm{y}=20.55-0.0362 \mathrm{x}+0.00078 \mathrm{x}^{2},
$$

with a minimum of $20.13^{\circ} \mathrm{C}$ for 1912 .

The average was $20.52^{\circ} \mathrm{C}$, with standard error of the mean equal to $0.14^{\circ} \mathrm{C}$. 
